\title{
Experimental Investigation of the Characteristics of the Electromagnetic Reverberation in the UWB bands
}

\author{
Aliou Bamba*, Wout Joseph senior member IEEE, \\ Emmeric Tanghe, Luc Martens member IEEE \\ Ghent University/iMinds, Department of Information Technology \\ Gaston Crommenlaan 8 box 201, B-9050 Ghent/Belgium \\ Email: aliou.bamba@intec.ugent.be
}

\author{
Davy Gaillot, Martine Lienard \\ Group TELICE, IEMN, University of Lille \\ Building P3, 59655 Villeneuve d'Ascq, France \\ Jose-Maria Molina-Garcia-Pardo, Maria-Teresa Martinez-Ingles \\ Universidad Politecnica de Cartagena, Cartagena 30202, Spain
}

\begin{abstract}
Room electromagnetics characteristics are investigated at Ultra-Wide Band (UWB) frequencies from 2 to $10 \mathrm{GHz}$ in an indoor environment for various $T x-R x$ positions. The reverberation time is found to be bandwidth independent and decreases as the frequency is increased. Besides, the reverberation ratio, i.e. the contribution of the diffuse fields to the total power, increases also with larger TxRx separation. A maximum value of the reverberation ratio of 0.6 is obtained for the investigated scenarios in the room.
\end{abstract}

Keywords: Reverberation time, reverberation ratio, diffuse multipath components, power density.

\section{INTRODUCTION}

$\mathrm{R}$ ECENT studies such as [1] have shown the importance of the diffuse multipath components (DMC) in realistic indoor environments in terms of its contribution to the total power density. The DMC is usually considered as the remainder of the measurements data after removing all possible specular paths. Hence, the DMC power can not be obtained directly from propagation mechanisms. Alternatively, the DMC total power density in a given indoor environment can rather be expressed as a function of its reverberation time [2], [3]; the reverberation time being the decay rate of the DMC and used in the room electromagnetics theory [2], [3].

The total power density of the DMC at any given location is highly dependent to the reverberation time and separation between the transceiver elements. The reverberation time has previously been investigated in office environments and in reverberation chamber as well [3], [4]. All the works mentioned above have been carried out at a single frequency, i.e., $1.4,2.3,3$, or $5.4 \mathrm{GHz}$. Up to now, no studies have addressed the characteristics of the reverberation time at higher frequencies with large bandwidths according to the authors knowledge. Here, the reverberation time characteristics are investigated from $2-10 \mathrm{GHz}$ as well as the total power of the DMC. The characteristics are namely the reverberation time dependency to both the bandwidth of the frequency since those are important features of modern telecommunication systems. The paper is organized as follows: the measurements scenario and the methods are summarized in Section II. The results are presented in Section III and conclusions are drawn in Section IV.

\section{Scenarios And Methods}

\section{A. Scenarios}

The measurements have been carried out in a laboratory of the Universidad Politecnica de Cartagena, Cartagena, Spain. The details of the environment, the measurement scenarios and the channel sounder settings can be found in [5]. The main settings are recalled here for the reader: $i$ ) the frequency is ranging between 2 and $10 \mathrm{GHz}$ with 2048 frequency points and ii) three Tx blocks were considered. Each block consists in $95 \mathrm{Tx}$ positions along a linear segment of $95 \mathrm{~cm}$ whereas the receiver $(\mathrm{Rx})$ occupied 8 positions along a linear segment of $70 \mathrm{~cm}$.

\section{B. Methods}

Each block (or segment of $95 \mathrm{~cm}$ ) is divided in 3 sub-blocks with 31 successive elements. The $8 \mathrm{Rx}$ positions are retained. The $\mathrm{Tx}$ and $\mathrm{Rx}$ are re-arranged so that the spatial correlation between two received signals in different sub-blocks is lower than 0.5 for the whole frequency band. This criterion is set to obtain spatially uncorrelated signals. Then, an averaged impulse response per subblock is obtained with 8 positions of the Rx and 31 positions of the Tx. This is sufficiently large to remove the small scale fading effects. The reverberation time is then determined from the averaged power delay profile (PDP) following the procedure detailed in [3]. One value of the reverberation time (which is characteristic of the room with respect to diffuse absorption) is further obtained by averaging the values for all the sub-blocks.

Once the reverberation time is determined, the received power due to the DMC is [2]:

$$
P_{d m c}=\frac{P_{0} c_{0} \tau}{V}\left(\frac{\lambda}{4 \pi}\right)^{2} \times e^{-\frac{d_{0}}{c_{0} \tau}} \times \eta_{p o l},
$$

where $\mathrm{P}_{0}, \mathrm{c}_{0}=3 \times 10^{8} \mathrm{~m} / \mathrm{s}, \mathrm{V}, \lambda, \mathrm{d}_{0}$, and $\eta_{\text {pol }}=0.5$ are the transmitted power, light speed in vacuum $(\mathrm{m} / \mathrm{s})$, room volume $\left(\mathrm{m}^{3}\right)$, wavelength $(\mathrm{m})$, Tx-Rx seperation $(\mathrm{m})$, and the factor accounting for the randomness of the DMC polarization, respectively. $\mathrm{P}_{d m c}$ has the same unit as $\mathrm{P}_{0}$ (Watt). Note the in (1), an omni-directional antenna with a unity gain is considered since complete diffuse fields are assumed. The total received power is calculated as follows:

$$
P_{\text {total }}=\sum_{i} P D P\left(\tau_{i}\right)
$$

Note that both (1) and (2) are calculated in a linear scale.

\section{RESULTS}

\section{A. Reverberation time as a function of the bandwidth}

Frequencies ranging from 2.5 to $9.5 \mathrm{GHz}$ with a step of $500 \mathrm{MHz}$ are investigated. The bandwidth varies from the narrow band to ultra wide band, i.e., from $100 \mathrm{MHz}$ up to $900 \mathrm{MHz}$ with a step of $50 \mathrm{MHz}$. Figure 1 presents the reverberation time as a function of bandwidth for different frequencies. Only selected frequencies are shown for the sake of clarity. The maximum relative standard deviation is about $2.2 \%$, clearly indicating that the reverberation time is bandwidth independent. This is attributed to the fact that the bandwidth does not affect the propagation phenomena but rather influences the small scale fading statistics since it determines the resolvable delay bin duration. However, the reverberation in the environment is due the large scale fading, which is attributed to physical phenomena such as the diffractions/shadowing, the scattering, free space attenuation, etc. 


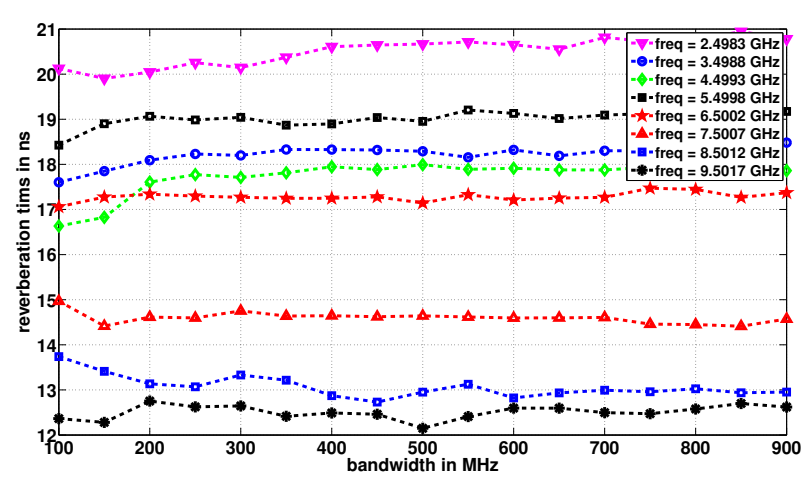

Fig. 1. Reverberation time as a function of the bandwidth for different frequencies.

\section{B. Reverberation time as a function of the frequency}

A bandwidth of $500 \mathrm{MHz}$ is now considered for the rest of this study as the reverberation time is bandwidth independent (Section III-A). Figure 2 shows the decrease of the reverberation time

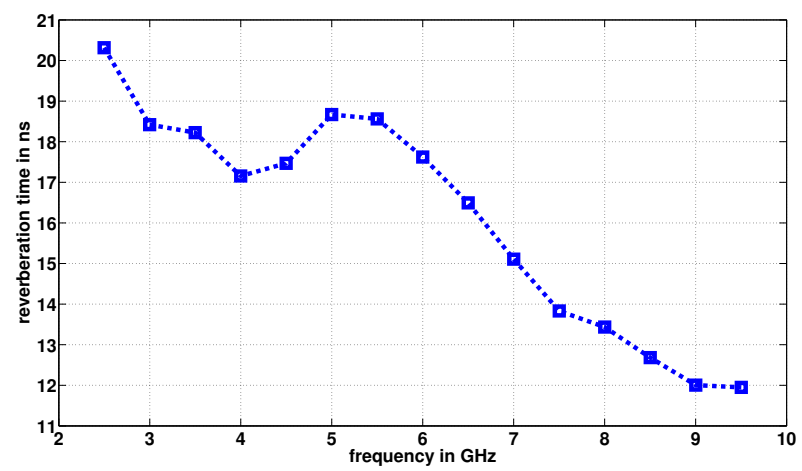

Fig. 2. Reverberation time as a function of the frequency. A bandwidth of $500 \mathrm{MHz}$ is considered. For a frequency, the reverberation time value is obtained by averaging 9 values from different spatial positions in the room.

as the frequency is increased. This shows that the energy at higher frequencies is fading faster away comparing to the energy at lower frequencies, which is caused by larger electrical distances at higher frequencies. The absorption coefficient of the building materials at higher frequencies contributes to the fast decay of the diffuse fields as well. However, a local maximum is observed in the 5-6 GHz region. Further investigations of the reverberation time frequency dependency should be performed in other indoor environments to verify whether this resonance phenomenon is due to the room dimensions, to the absorption properties of the building materials, or to the reverberation itself.

\section{Diffuse power contribution in the total power}

It might be helpful to quantify the impact of the DMC when designing a wireless system. The DMC impact in terms of its contribution to the total power could be used - if not negligible - to increase the system performance or to determine the possible health effects due to the diffuse fields. The term reverberation ratio is here used as in [6] to define the ratio of the DMC power and the total power.

$$
r=\frac{P_{d m c}}{P_{\text {total }}},
$$

where $\mathrm{P}_{d m c}$, and $\mathrm{P}_{\text {total }}$ are the power due to the diffuse fields and the total power, respectively. Figure 3 shows the reverberation ratio (r) as a function of the frequency and for different Tx-Rx separations. For a given frequency, the reverberation ratio becomes larger as the

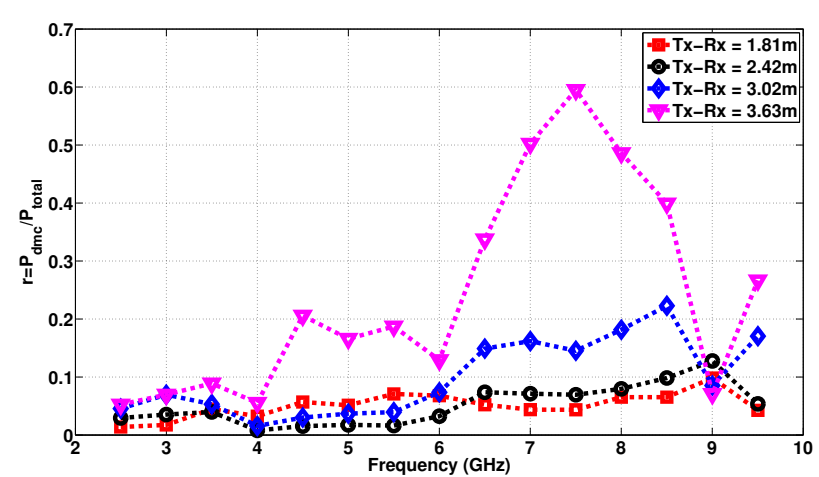

Fig. 3. Reverberation ratio as a function of the frequency. A bandwidth of $500 \mathrm{MHz}$ is considered.

Tx-Rx separation increases. This experimental observation agrees with [6], where it is found that the reverberation ratio as a function of the distance shows a resonance phenomenon occurring from a certain Tx-Rx distance. However, this resonance phenomenon could not have been investigated because of the short Tx-Rx separations due to the room dimensions - during our measurement campaign. It is noteworthy to mention that both the DMC power and the total power decrease as the frequency increases. An increasing reverberation ratio shows that the DMC power decreases slower than the total power does.

\section{CONCLUSIONS}

The frequency and bandwidth dependence of the electromagnetic reverberation time is experimentally investigated in an indoor environment. The results demonstrate that it is bandwidth independent and decreases as a function of frequency, showing that the diffuse fields fade at a faster rate at higher frequencies. The contribution of the diffuse fields to the total power is not significant $(<10 \%)$ at short Tx-Rx separations while it becomes prominent at large Tx$\mathrm{Rx}$ separations in agreement with the literature. A local maximum is observed in the reverberation ratio as a function of the frequency. However, this observation has to be confirmed with more extensive measurements and has to be supported with theoretical backgrounds. This will be part of future works on this topic.

\section{REFERENCES}

[1] Juho Poutanen, Jussi Salmi, Katsuyuki Haneda, Veli-Matti Kolmonen, and Pertti Vainikainen. Angular and Shadowing Characteristics of Dense Multipath Components in Indoor Radio Channels. IEEE Transactions on Antennas and Propagation, 59: pages 245-253, 2011.

[2] Jørgen Bach Andersen, Kin Lien Chee, Martin Jacob, Gert Frølund Pedersen, Thomas Kürner. Reverberation and Absorption in an Aircraft Cabin with the Impact of Passengers. IEEE Transactions on Antennas and Propagation, 60 (5): pages 2472-2480, 2012.

[3] A. Bamba, W. Joseph, J. B. Andersen, E. Tanghe, G. Vermeeren, D. Plets, J. O. Nielsen, and L. Martens. Experimental Assessment of Specific Absorption Rate Using Room Electromagnetics. IEEE Transactions on Electromagnetic Compatibility, 54 (4): pages 747-757, 2012.

[4] Aliou Bamba, Davy Gaillot, Emmeric Tanghe, Wout Joseph, Martine Lienard, Luc Martens. Assessing Human Whole-Body Specific Absorption Rate For diffuse Exposure From Reverberation Chamber Measurements. submitted to IEEE Transactions on Electromagnetic Compatibility (accepted under revision conditions), 2013.

[5] Maria-Teresa Martinez-Ingles, Jose-Maria Molina-Garcia-Pardo, Jose-Victor Rodriguez, Juan Pascual-Garcia, and Leandro Juan-Llacer. Experimental Comparison between Centimetre- and Millimetre- Wave Ultra - Wideband Radio Channels. submitted to IET Microwaves, Antennas \& Propagation (accepted under revision conditions), 2014.

[6] Gerhard Steinbock, Troels Pedersen, Bernard Henri Fleury, Wei Wang, and Ronald Raulefs. Distance Dependent Model for the Delay Power Spectrum of In-room Channels. IEEE Transactions on Antennas and Propagation, 61 (8): pages $4327-$ 4340, 2013. 